\title{
A deep dive into the left ventricle
}

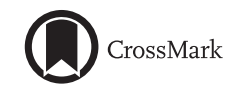

\author{
Hiroo Takayama, MD, PhD, ${ }^{\mathrm{a}, \mathrm{b}}$ Shepard D. Weiner, MD, ${ }^{\mathrm{a}, \mathrm{c}}$ and Emile A. Bacha, $\mathrm{MD}^{\mathrm{a}, \mathrm{b}}$ \\ From the ${ }^{\mathrm{a}}$ Hypertrophic Cardiomyopathy Center, ${ }^{\mathrm{b}}$ Division of Cardiac, Thoracic and Vascular Surgery, Depart- \\ ment of Surgery, and ${ }^{\mathrm{c} D i v i s i o n}$ of Cardiology, Department of Medicine, Columbia University Medical Center/ \\ NewYork-Presbyterian, New York, NY. \\ Disclosures: Authors have nothing to disclose with regard to commercial support. \\ Received for publication Nov 2, 2017; accepted for publication Nov 9, 2017; available ahead of print Dec 1, 2017. \\ Address for reprints: Emile A. Bacha, MD, Division of Cardiac, Thoracic and Vascular Surgery, NewYork- \\ Presbyterian/Columbia University Medical Center, 3959 Broadway, Rm 274, New York, NY 10032 (E-mail: \\ eb2709@cumc.columbia.edu). \\ J Thorac Cardiovasc Surg 2018;155:2094-5 \\ $0022-5223 / \$ 36.00$ \\ Copyright $(2) 2018$ by The American Association for Thoracic Surgery \\ https://doi.org/10.1016/j.jtcvs.2017.11.012
}

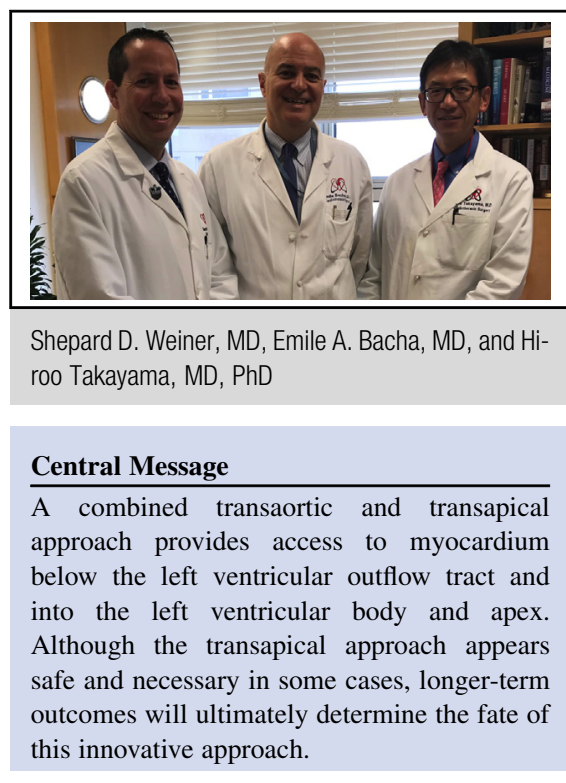

See Article page 2096
The group from Mayo Clinic, the leading hypertrophic cardiomyopathy (HCM) program, has championed septal myectomy and contributed substantially to the development of the current surgical standards. In this issue of the Journal, ${ }^{1}$ they report another interesting surgical modification of this procedure, a combined transaortic and transapical approach for "complex" HCM. Complex HCM was defined as any HCM not confined to the subaortic region, namely any long-segment HCM with midventricular obstruction (MVO) (either from the get-go or after resection), or apical HCM, with cavity obliteration, reduced LV volume, and diastolic dysfunction. The transapical approach was introduced in an ambitious attempt to relieve MVO and/or to augment diastolic filling. Besides 2 inhospital mortalities among 86 patients, all others appeared to have enjoyed satisfactory resolution of the intracavitary gradient with excellent midterm outcomes. Complication rates were low.

The question then becomes: Should this procedure be added to the armamentarium of every surgeons performing septal myectomies?

Looking into the specific details of this patient population, only $3.4 \%$ required this approach among a total of 2508 patients in this tertiary referral HCM program. It would therefore be extremely rare for cardiac surgeons in low- to medium-volume HCM programs to encounter the 2 specific situations in which this procedure is truly necessary. Moreover, in addition to the increased surgical complexity of adding a ventriculotomy to transaortic septal myectomy, even in these experienced hands, $25 \%$ patients required a second or more aortic crossclamping for further myectomy, indicating that need of additional myocardial ischemia is almost a part of their standard practice in this cohort. The rarity and complexity might negatively influence the reproducibility of this procedure in smaller programs and could potentially harm patients. In contrast, there is a small subset of "typical" HCM patients who have residual postoperative MVO with symptoms. They are frequently in need for a reoperation. That subset of patients would be greatly helped by a more aggressive but definitive first operation using this combined approach.

Only one half of the cohort had the diagnosis of MVO before they underwent cardiopulmonary bypass, and the reported cases are a mixture of MVO and apical HCM. The established role of septal myectomy for HCM is to relieve left ventricular outflow tract obstruction, and relief of MVO fits within this spectrum; however, surgical therapy with myectomy for diastolic dysfunction in apical HCM has distinctly different goals. ${ }^{1}$ Because its efficacy is yet to be determined, widespread implementation of this practice (apical myectomy) might be premature, and we await longer-term data on improvement of diastolic left ventricular function and quality of life.

More important than achieving technical success, which, when measured by disappearance of the left ventricular outflow tract gradient, the Mayo surgeons seems to have achieved, the clinical efficacy of the procedure is yet to be determined fully. It appears that neurologic events were the most common. Given that the primary goal of septal myectomy is to improve the quality of life and not necessarily the survival, disabling complications are to be avoided at all cost. Although $97.6 \%$ of survivors experienced functional improvement, detailed follow-up information beyond 30 days was available in only roughly one half of the patients. Although the present study demonstrated short-term safety and feasibility, there are a number of 
long-term concerns to be addressed, such as left ventricular dysfunction, arrhythmia, and pseudoaneurysm formation at the apical incision site. Again, any significant morbidity, short-term or long-term, defeats the purpose of this surgery.

Experts in HCM claim a shortage of myectomy surgeons. ${ }^{2}$ The imperative first step for any surgeon is to master the standard transaortic extended septal myectomy. The Mayo group has shown that the transapical approach, done for the right reasons in a large-volume center, is safe. Whether it should be widely used is another issue altogether, and we eagerly await their long-term data. In the meantime, for surgeons who deal with HCM frequently, this procedure is a great addition.

\section{References}

1. Hang D, Schaff HV, Ommen SR, Dearani JA, Nishimura RA. Combined transaortic and transapical approach to septal myectomy in patients with complex hypertrophic cardiomyopathy. J Thorac Cardiovasc Surg. 2018;155: 2096-102.

2. Maron BJ, Dearani JA, Maron MS, Ommen SR, Rastegar H, Nishimura RA, et al Why we need more septal myectomy surgeons: an emerging recognition. J Thorac Cardiovasc Surg. 2017; 154:1681-5. 